\title{
L- and M-Cone isolating ERGs: LED versus CRT stimulation
}

\author{
I.J. MURRAY, ${ }^{1}$ J. KREMERS, ${ }^{2}$ AND N.R.A. PARRY ${ }^{3}$ \\ ${ }^{1}$ Faculty of Life Sciences, University of Manchester, Manchester, United Kingdom \\ ${ }^{2}$ Department of Ophthalmology, University of Erlangen-Nuremberg, Erlangen, Germany \\ ${ }^{3}$ Vision Science Centre, Manchester Royal Eye Hospital, Manchester, United Kingdom
}

(RECEIVED October 15, 2007; ACCEPTEd February 11, 2008)

\begin{abstract}
Using double silent substitution, it is possible to generate L-cone and M-cone isolating electroretinograms (ERGs) on a CRT. A major limitation of the technique is that the depth of modulation of cone classes is limited by the restricted luminance of the phosphors and their spectral overlap. To address this problem we have ported the technique to a four-color LED Ganzfeld stimulus (Diagnosis ColorDome). This allows higher retinal illuminances, higher contrasts, and triple silent substitution. With careful control over the retinal area stimulated, we show that the same data can be recorded from both CRT and LED stimuli when luminance, size and cone contrast are kept constant. Importantly, the different temporal profiles of the two devices do not influence the ERG amplitude and phase plots. We present data over a much wider range of luminances (up to about 10000 trolands) and contrasts with the LED stimulator than previously reported with CRT screens. We conclude that the close resemblance between data obtained with an LED stimulator and with a CRT screen indicate that the differences have a purely physiological origin.
\end{abstract}

Keywords: Electroretinograms, L-cones, M-cones, LED, CRT

\section{Introduction}

Double silent substitution can be used to record electroretinograms (ERGs) selectively from human L- and M-cones (Kremers et al., 1999; Brainard et al., 1999). When computer monitors are employed, a wide range of spatially varying stimuli can be generated so as to investigate the link between retinal area stimulated and proportions of L- and M-cone responses (Murray et al., 2004). Major disadvantages of conventional monitors, however, are that the spectral overlap in the emission spectra of the TV phosphors limits the available depth of modulation of cone contrast and that the maximum luminance is quite low.

In order to extend the range of stimulus parameters, we have transferred the silent substitution technique to a commercial four-color LED Ganzfeld stimulus. The high luminance and relatively narrow spectral bandwidth of the LEDs allows much higher retinal illuminances and higher contrasts to be obtained and of course, triple silent substitutions are feasible. One important question to be addressed is whether the sharper temporal profile of the LEDs would influence the generation of the ERGs. Hence, the motivation for the experiments was to establish that, provided exactly the same area and luminance were used, the signal amplitude and phase obtained with the two stimuli would remain unchanged.

Address correspondence and reprint requests to: I. J. Murray, Faculty of Life Sciences, University of Manchester, Moffat Building, P.O. Box 88, Manchester M60 1QD, UK. E-mail: ian.j.murray@manchester.ac.uk

\section{Materials and methods}

\section{CRT Stimulus}

Circular (diameter $21.8^{\circ}$ ), L-only, M-only, and L + M stimuli (when $\mathrm{L}$ cones and $\mathrm{M}$ cones were stimulated in phase) were sinusoidally modulated at $30 \mathrm{~Hz}$ on a Sony GDM F520 high resolution monitor, viewed at a distance of $20 \mathrm{~cm}$. The frame rate was $120 \mathrm{~Hz}$. The stimuli were generated using a VSG2/4 card (Cambridge Research Systems, Rochester, UK) and our own software. Mean luminance was $66 \mathrm{~cd} / \mathrm{m}^{2}$ (Red:Green:Blue luminance ratio $=20: 40: 6$ ). The maximum attainable contrast for $\mathrm{L}$ and $\mathrm{M}$ cones in isolation was $24.7 \%$ and $31.3 \%$, respectively, and $76.8 \%$ for each cone when they were stimulated simultaneously. S-cone contrast was negligible. Mean luminance of the modulated part of the screen was $66 \mathrm{~cd} \cdot \mathrm{m}^{-2}$. The surround luminance was 33 $\mathrm{cd} \cdot \mathrm{m}^{-2}$. See Kremers et al. (1999) for a detailed description of the methodology. Briefly, cone isolation was achieved by multiplying the energy spectra of the three CRT phosphors and the three cone-photoreceptor classes and calculating combinations that produced silent substitution. The monitor was calibrated using a SpectraScan PR650 spectroradiometer (PhotoResearch Inc, Chatsworth, USA).

\section{LED stimulus}

The same double silent substitution technique was used to generate $\mathrm{L}, \mathrm{M}$, and $\mathrm{L}+\mathrm{M}$ stimuli on a commercially available Ganzfeld stimulus (ColorDome; Diagnosis LLC, Lowell, MA). This contains 
four LED types, with the following peak wavelengths ( \pm bandwidth): $635 \pm 10 \mathrm{~nm}$ ("red"), $595 \pm 8 \mathrm{~nm}$ ("amber"), $514 \pm 20 \mathrm{~nm}$ ("green"), and $465 \pm 10 \mathrm{~nm}$ ("blue"). The amber LED was not used in these experiments as we wished to make a direct comparison with the CRT device. The software used to calculate coneisolating stimuli for the CRT stimulus was used for the LED stimulus, substituting the CRT phosphor spectra with those of the red, green, and blue LEDs. The output of this program was mean luminance, modulation depth, and phase for each LED type. These were used to produce script files to generate R, G, and B luminance profiles, with $1 \mathrm{~ms}$ resolution. Calibration was again performed with the PR650. Theoretical maximum luminances for each LED in isolation were $1971 \mathrm{~cd} \cdot \mathrm{m}^{-2}$ (red), $3584 \mathrm{~cd} \cdot \mathrm{m}^{-2}$ (green), $457 \mathrm{~cd} \cdot \mathrm{m}^{-2}$ (blue), and $1305 \mathrm{~cd} \cdot \mathrm{m}^{-2}$ (amber), but for thermal stability luminances were restricted to $10 \%$ of these values. Maintaining the same RGB ratio of 20:40:6, this gave a maximum luminance of $330 \mathrm{~cd} \cdot \mathrm{m}^{-2}$, and maximum cone contrasts of $\mathrm{L}=0.34, \mathrm{M}=0.49$, and 0.813 each when they were stimulated together $(\mathrm{L}+\mathrm{M}$ condition). In the experiments where the same stimuli were presented on both devices, the Ganzfeld was masked with white card to give a visible disk of $21.8^{\circ}$ at $20 \mathrm{~cm}$ viewing distance. The luminance of the rest of the card was approximately $30 \mathrm{~cd} \cdot \mathrm{m}^{-2}$. The device contains an infrared camera to allow direct observation of the pupil. This is particularly important when high luminances are employed when the photic flinch reflex (Murray et al., 2002) can reduce retinal illumination through lid occlusion. We also present here preliminary data from an LED experiment in which the whole luminance range was employed; in this case, the Ganzfeld was used conventionally to give full-field stimulation.

\section{Electroretinograms}

Electroretinograms (ERGS) were recorded using two different active electrodes, a corneal silver/nylon thread (DTLplus, Diagnosis LLC, Lowell, MA) draped across the limbus, and a skin-mounted gold-impregnated Mylar film electrode (Burden Neuroscience, Bristol, UK) attached to the lower eyelid, just below the lash line, using electrode gel. Reference for both electrodes was a $9 \mathrm{~mm} \mathrm{Ag} / \mathrm{AgCl}$ EEG electrode (Biosense Medical, Chelmsford, UK) mounted at the ipsilateral outer canthus, and a similar earth electrode was placed on the forehead. Signals were amplified using a Grass 15A94 amplifier with gain of 20,000 $\times$ and bandwidth of 3-100 Hz. Data were recorded using a CED1401 plus interface running Signal 2.0 acquisition software (Cambridge Electronic Designs, Cambridge, UK). Signals were acquired at $1024 \mathrm{~Hz}$, in epochs of $4 \mathrm{~s}$. At least 32 sweeps (4096 points) were recorded for each condition. Post acquisition, the data were subjected to a discrete Fourier transform to extract the first harmonic $(30 \mathrm{~Hz})$, together with the $29 \mathrm{~Hz}$ response as a measure of noise. Second harmonic data are not included in the present analysis. The data presented are vector averages of individual sweeps.

\section{Stimulus timing}

One of the fundamental differences between the two devices is that luminance changes with the ColorDome are instantaneous whereas the CRT paints the stimulus over the duration of a single frame. To allow valid comparisons of ERG phase using the two devices, it was important not to confuse phase effects caused by triggering differences with those that might be due to the different temporal characteristics of the stimuli themselves. To measure this, we coupled a photo-multiplier (PMT) to the CED1401 plus and used Fourier analysis to measure the phase of the luminance modulation of each device. In the case of the LED stimulus and at $30 \mathrm{~Hz}$, the measured phase was $90^{\circ}$. This is equivalent to zero delay because the FFT computation is based on the cosine of the response. This was confirmed by stimulating at different rates, when PMT phase remained constant at $90^{\circ}$.

To measure the CRT, a single pixel was modulated at the top left-hand corner of the CRT (closest to the trigger). At $30 \mathrm{~Hz}, \mathrm{PMT}$ phase was $-28^{\circ}$, indicating that the earliest luminance modulation on the screen lagged behind the LED device by $118^{\circ}$. This was due to a combination of software and hardware delays, and meant that, to directly compare ERG phases with the two devices, we should add $118^{\circ}$ to the ERG phase when using the CRT. At $120 \mathrm{~Hz}$, the screen refreshes every $8.34 \mathrm{~ms}$, and much of this time is spent painting the screen. Placing the single pixel at top left, middle, and bottom right of the screen gave phases of $-28^{\circ},-64^{\circ}$, and $-98^{\circ}$, respectively. Hence, it took $70^{\circ}$ (i.e., $6.48 \mathrm{~ms}$ ) to paint the screen, with the rest of the $8.34 \mathrm{~ms}$ being taken up with front and back porches and fly-back. Finally, as an index of the temporal smearing inherent in CRT displays, we presented the $8 \mathrm{~cm}$ disk used as the $21.8^{\circ}$ circular stimulus, at the top left, middle, and bottom right of the screen. We recorded the PMT response using an integrating sphere, and measured phases of $-40^{\circ},-62^{\circ}$, and $-84^{\circ}$.

\section{Subjects}

Data from three male subjects are presented here, two authors (IJM and JK, aged, respectively, 56 and 47) and a naïve subject (HJT aged 24). All were color normal. All responses were recorded monocularly (right eye) through a dilated pupil. Dilatation and lid position were carefully monitored during every sweep to ensure that retinal illuminance was constant for each subject and throughout each data collection session.

\section{Results}

Fig. 1 illustrates corneal and skin ERG amplitude (top panel), and phase (lower panel) obtained with the LED and the CRT stimulus for observer JK. The figure also contains a calibration bar to aid conversion from phase to delay in ms. It is evident that there is no difference in amplitude between the LED and CRT stimuli.

In order to discount any differences in trigger time, $118^{\circ}$ was added to the phase of the CRT response (see Materials and methods) so that the reference point for ERG phase was the top left corner of the screen. Note that for the DTL electrode the CRT top left location phase is $-76^{\circ}$, compared with $-56^{\circ}$ for the LED. Thus, the CRT produces a small lag in phase of $20^{\circ}$. This equates to a delay of $1.87 \mathrm{~ms}$, which can be attributed to temporal smearing. The phase shift between the top left and bottom right locations is $45^{\circ}$ and this is very close to that measured with the PMT $\left(44^{\circ}\right)$. The systematic difference between skin and DTL electrodes is $16^{\circ}$, which equates to about $1.5 \mathrm{~ms}$. In our lab, we have found the signal:noise ratio with skin electrodes to be superior where there is the potential for small eye-movement artifacts (e.g., with very close screen viewing), although in this paper we concentrate on the corneal electrode.

Fig. 2 shows ERG amplitudes for two subjects comparing L-only, M-only, or L + M stimulation with the LED stimulus (dark bars) and the CRT (pale bars). Note that $\mathrm{L}+\mathrm{M}$ stimulation is approximately the same in amplitude for the two subjects (around $0.8 \mu \mathrm{V}$ ), but the proportions of L-only versus M-only 

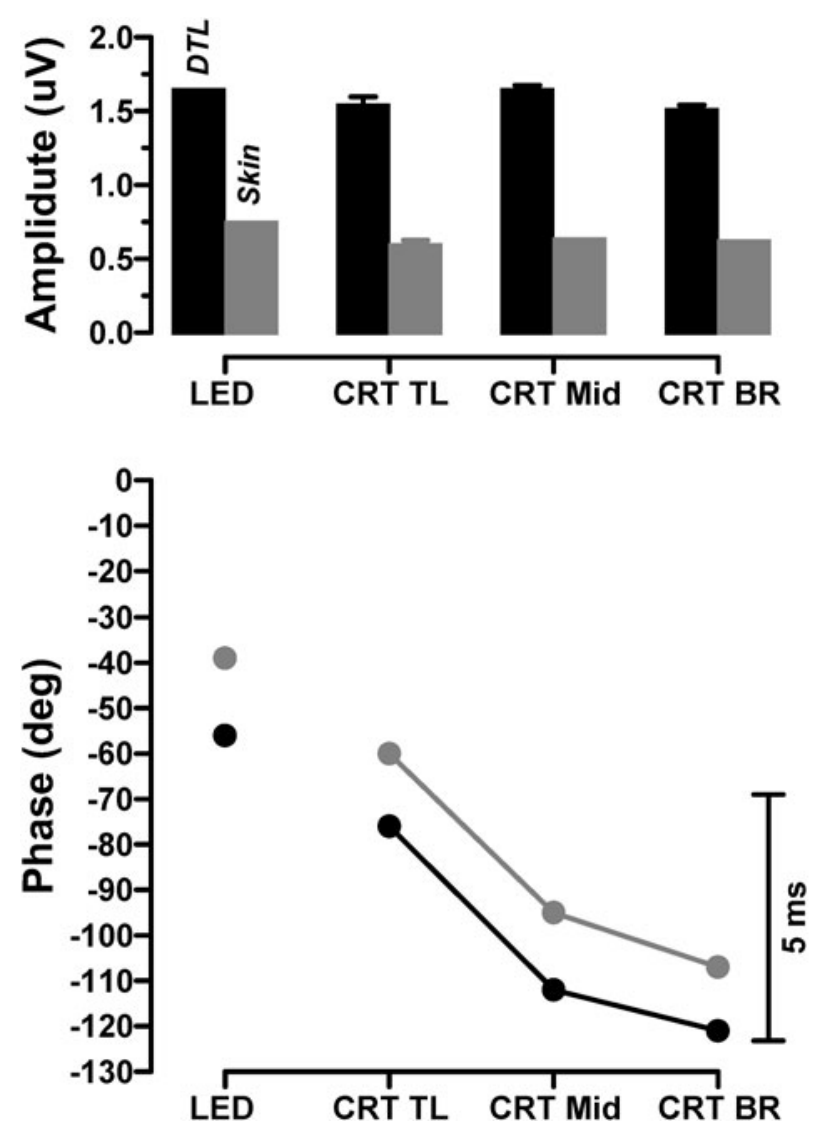

Fig. 1. Comparison of LED and CRT ERGs to a $21.8^{\circ}$ stimulus in which $\mathrm{L}$ and $\mathrm{M}$ cones were modulated in phase with equal cone contrast of 0.34 . Three results are shown for the CRT data, with the stimulus located at the top left (TL), the middle (Mid), and the bottom right (BR) of the monitor. Results for skin (Burden) and corneal (DTL) electrodes are shown. Top panel depicts amplitude, with 95\% CIs. Phase is shown in bottom panel. The calibration bar is provided to aid conversion between phase and delay for the $30 \mathrm{~Hz}$ presentation rate. The conversion to delay assumes that phase changes are within a single phase cycle (i.e, less than $360^{\circ}$ ). Subject JK.

are conspicuously different, reflecting the different $\mathrm{L} / \mathrm{M}$ ratios of these observers. In a separate psychophysical study, we have estimated that IJM's L:M ratio is approximately twice that of HJT's (6.4 versus 2.8)

Fig. 3 presents amplitude versus contrast (left panels) and amplitude versus retinal illuminance (right panels) for observers HJT (top) and IJM (bottom). LED data are dark symbols and CRT data are pale symbols. The range of contrast comparisons is restricted because the CRT has a smaller contrast range than the CRT stimulus. It is clear however that the CRT and the LED stimulus give comparable amplitudes and that the contrast gains obtained with the two devices are similar.

In the right-hand panels, ERG amplitudes are plotted against logarithm of retinal illuminance for the LED stimulus, viewed as a ganzfeld. L-only and M-only data are depicted as dark and pale triangles, respectively, and $\mathrm{L}+\mathrm{M}$ measured are filled circles. Stiles-Crawford effects are not included in the calculation of retinal illumination. The equivalent calculated data $(\mathrm{L}+\mathrm{M}$ calc), obtained by vector addition of the L- and M-specific signals, are open circles. It is interesting to compare the cone specific amplitudes for the two observers. Note that M-cones (dark triangles)
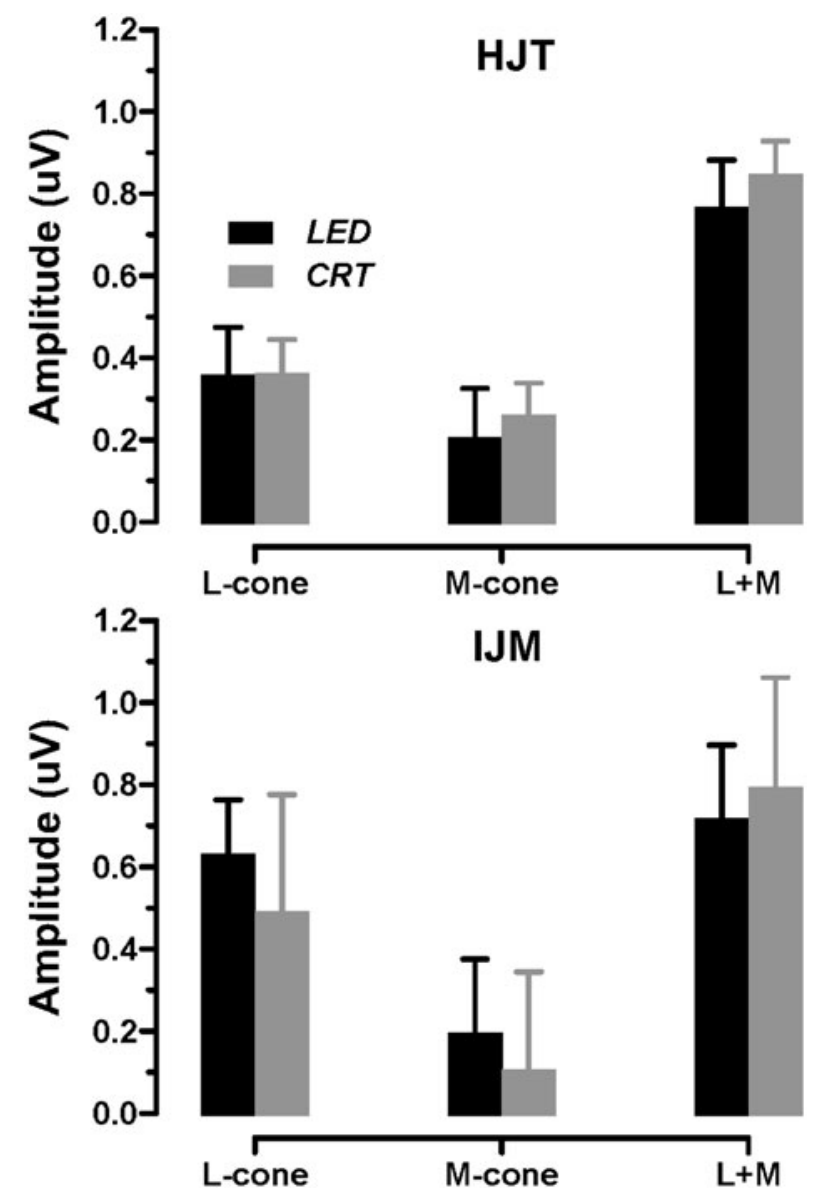

Fig. 2. Comparison of ERGs recorded with LED and CRT stimulation. Stimulus was a $21.8^{\circ}$ disk in which either $\mathrm{L}$ or $\mathrm{M}$ cones were modulated at 0.34 contrast, or both were modulated together. Two subjects are shown, HJT, whose LM ratio is 2.8, and IJM, whose LM ratio is 6.4.

generate a measurable response across the full range of retinal illuminances for observer HJT (top panel) but in the case of observer IJM, the M-cone response remains in the noise (pale circles) for the three lower illuminances and emerges from the noise only above 1000 td. For IJM the L-cone responses are slightly greater in amplitude than those for HJT.

\section{Discussion}

The objective of these experiments was to test a technique for generating cone-specific ERGs using an LED-based stimulus. The method was compared with previous studies which used a CRT monitor (Kremers et al., 1999; Murray et al., 2004). We used the same stimulus diameter, a range of stimulus contrasts, and two subjects with markedly different $\mathrm{L} / \mathrm{M}$ ratios according to heterochromatic flicker photometry. There are no systematic differences between ERG signals generated by the two stimuli providing that care be taken to ensure the conditions are constant between the two devices.

As stated above, although a ganzeld is not as spatially versatile as the CRT, the latter is limited by the spectral overlap of its phosphors and the maximum luminance of the red, green, and blue guns. This limitation is important because some subjects generate 


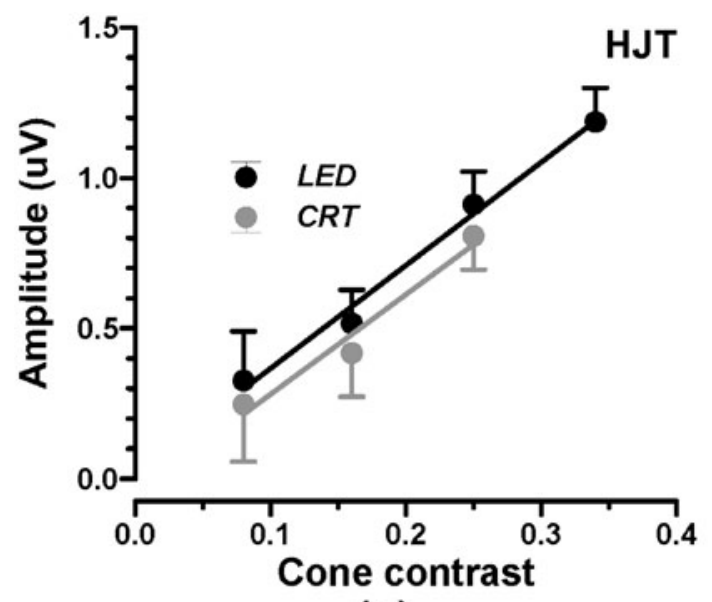

(a)

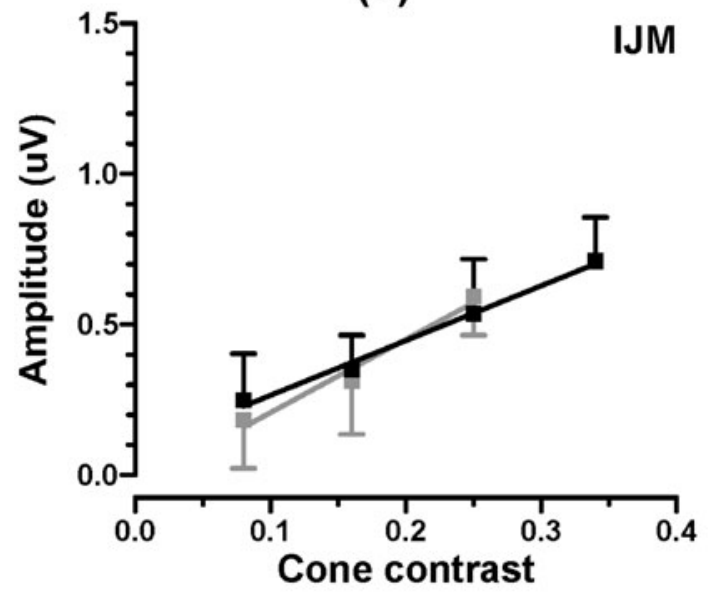

(b)

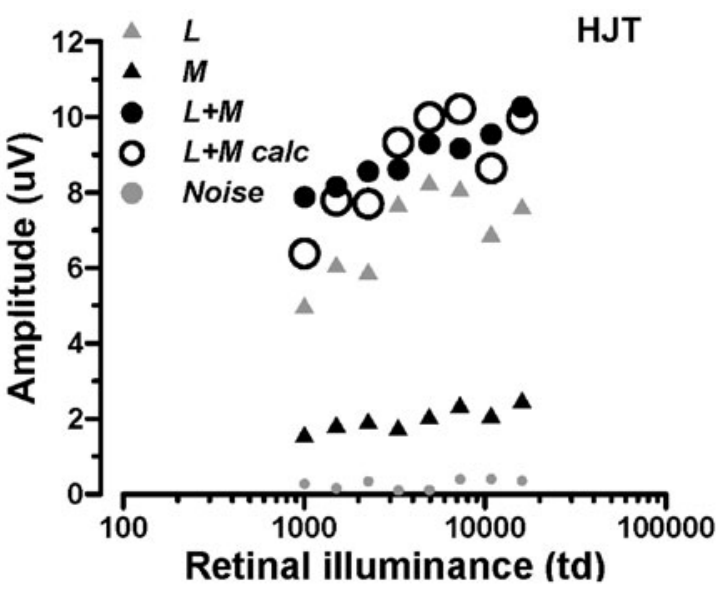

(c)

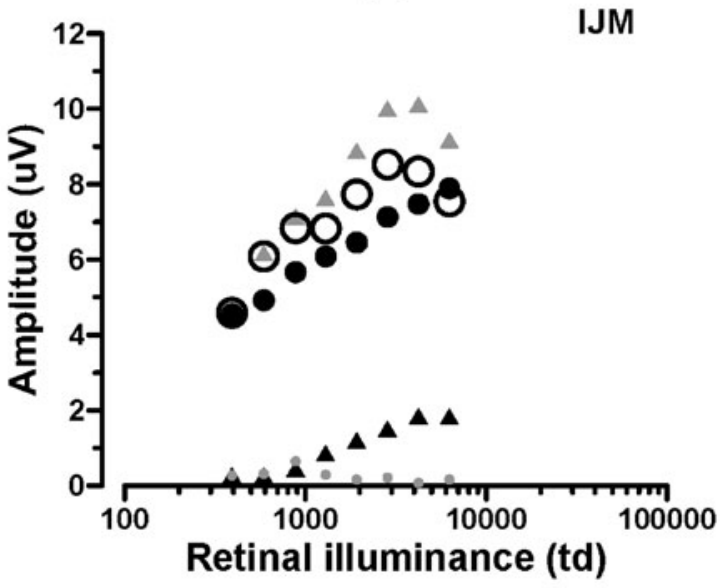

(d)

Fig. 3. (a and b) L + M ERGs as a function of cone contrast in two subjects, comparing LED and CRT stimulation. L and M cones modulated in-phase. Error bars are 95\% CIs. (c and d) Show ERGs to L-only, M-only, and L + M stimulation for a range of luminances on the LED stimulus and cone contrasts of 0.34 . The open circles ( $\mathrm{L}+\mathrm{M}$ calc) show the results of vector addition of the $\mathrm{L}$ and $\mathrm{M}$ responses.

particularly weak M-cone ERGs. This may be due to a low number of M-cones, but it may also be explained by small shifts in $\lambda$ max of the L- and M-cone spectra. This would change the calculation of the silent substitution stimuli. In either case, increasing retinal illumination should enable M-cone activity to be detected and subsequently enable L- and M-cone responses to be recorded over a wider dynamic range than previously. The data for observer IJM shown in Fig. 3 illustrate this point; M-cone responses are largely absent for the range of luminances available from the CRT. M-cone activity is reliably detected only when the retinal illumination is greater than around $1000 \mathrm{td}$.

In Fig. 3, there is a hint that the L-cone luminance response saturates at high luminance. This is seen for both subjects presented here, but also in other studies in our laboratory. It may be an important aspect of the electrogenesis of cone-specific signals, because it may represent a breakdown in the additivity of $\mathrm{L}$ and $\mathrm{M}$ cone specific signals. It has previously been shown that for the most part additivity holds for L and M cone ERGS. That is, as seen in Fig. 3, the linear addition of L-specific and M-specific signals matches the signal obtained when $\mathrm{L}$ and $\mathrm{M}$ cones are stimulated simultaneously. This so-called linearity principle holds only when vector addition is used, that is the calculation must take account of the phase difference between the photoreceptor types. This has been explicitly tested (Kremers et al., 1999; Murray et al., 2004; Brainard et al., 1999) and with a few exceptions found to be valid. It does however assume that the signals from the two cone classes combine linearly and that they contribute equally to the postreceptoral site where they are generated. It is not known whether the phase differences, which differ remarkably between subjects, are associated with receptor physiology or differences in postreceptoral pathways. To investigate, these fundamental aspects of cone ERGs it will be necessary to extend the range of parameters beyond those offered by a CRT-based stimulus and this is possible with the device described in this report.

To conclude, we have transferred the method of recording L-cone and M-cone specific ERGs to a system based on LEDs. Data are presented for three color-normal observers. It is apparent from this preliminary investigation that the data from the LED device match those from a CRT monitor in terms of retinal illuminance and cone contrast and for observers with different $\mathrm{L} / \mathrm{M}$ ratios. Further validation of the technique awaits data from dichromats. 
LED-based systems allow the investigation of a wider range of stimulus parameters than those based on CRTs, but they are less complex than Maxwellian-view-based methods. CRT-based methods may not be readily available in the future and so LED systems may be valuable in understanding clinical problems. They will undoubtedly be useful in resolving some of the remaining puzzles regarding the physiological basis of the flicker ERG.

\section{Acknowledgments}

We would like to thank the referees for carefully reading the manuscript and making some valuable comments. J.K. received support from the German Research Council, grant KR 1317/9-1.

\section{References}

Brainard, D.H., Calderone, J.B., Nugent, A.K. \& Jacobs, G.H. (1999). Flicker ERG responses to stimuli parametrically modulated in color space. Investigative Ophthalmology \& Visual Science 40, 2840-2847.

Kremers, J., Usui, T., Scholl, H.P.N. \& Sharpe, L.T. (1999). Cone signal contributions to electroretinograms in dichromats and trichromats. Investigative Ophthalmology and Visual Science 40, 920-930.

Murray, I.J., Parry, N.R. A., Kremers, J., Stepien, M. \& Schild, A. (2004). Photoreceptor topography and cone-specific electroretinograms. Visual Neuroscience 21, 231-235.

Murray, I.J., Plainis, S. \& Carden, D. (2002). The ocular stress monitor: A new device for measuring discomfort glare. Lighting Research and Technology 34, 231-242. 